\title{
Rapid and sensitive identification of omicron by variant-specific PCR and nanopore sequencing: paradigm for diagnostics of emerging SARS-CoV-2 variants
}

\author{
Christopher Dächert ${ }^{1} \cdot$ Maximilian Muenchhoff ${ }^{1,2} \oplus \cdot$ Alexander Graf $^{1,3}\left[\right.$ [D $\cdot$ Hanna Autenrieth ${ }^{1} \cdot$ Sabine Bender ${ }^{1} \cdot$ \\ Helga Mairhofer ${ }^{1} \cdot$ Paul R. Wratil $^{1}$ (D) Susanne Thieme ${ }^{3} \cdot$ Stefan Krebs $^{3}$ (D) - Natascha Grzimek-Koschewa ${ }^{1,2}$. \\ Helmut Blum ${ }^{3}$ (D) Oliver T. Keppler ${ }^{1}$ (1)
}

Received: 4 January 2022 / Accepted: 8 January 2022 / Published online: 21 January 2022

(c) The Author(s) 2022

\begin{abstract}
On November 26, 2021, the World Health Organization classified B.1.1.529 as a severe acute respiratory syndrome coronavirus 2 (SARS-CoV-2) variant of concern (VoC), named omicron. Spike-gene dropouts in conventional SARS-CoV-2 PCR systems have been reported over the last weeks as indirect diagnostic evidence for the identification of omicron. Here, we report the combination of PCRs specific for heavily mutated sites in the spike gene and nanopore-based full-length genome sequencing for the rapid and sensitive identification of the first four COVID-19 patients diagnosed in Germany to be infected with omicron on November 28, 2021. This study will assist the unambiguous laboratory-based diagnosis and global surveillance for this highly contagious $\mathrm{VoC}$ with an unprecedented degree of humoral immune escape. Moreover, we propose that specialized diagnostic laboratories should continuously update their assays for variant-specific PCRs in the spike gene of SARS-CoV-2 to readily detect and diagnose emerging variants of interest and VoCs. The combination with established nanopore sequencing procedures allows both the rapid confirmation by whole genome sequencing as well as the sensitive identification of newly emerging variants of this pandemic $\beta$-coronavirus in years to come.
\end{abstract}

Keywords SARS-CoV-2 $\cdot$ VOC $\cdot$ VOC-PCR $\cdot$ Nanopore $\cdot$ Omicron $\cdot$ Melting curve analysis

\section{Introduction}

Already in the last weeks of December 2021, omicron has become the dominant variant of concern ( $\mathrm{VoC})$ in many countries, including Great Britain, France, Denmark,

Edited by Matthias J. Reddehase.

Christopher Dächert, Maximilian Muenchhoff and Alexander Graf contributed equally.

Oliver T. Keppler

keppler@mvp.lmu.de

1 Max von Pettenkofer Institute \& Gene Center, Virology, National Reference Center for Retroviruses, LMU München, Pettenkoferstr. 9a, 80336 Munich, Germany

2 German Center for Infection Research (DZIF), Partner Site Munich, Munich, Germany

3 Laboratory for Functional Genome Analysis, Gene Center, LMU München, Munich, Germany
Greece, Spain, Portugal, and the US [1] with numbers of new infections reaching all-time highs. Earlier VoCs were characterized either by an increased ability for transmission in the population [VoCs alpha (B.1.1.7) and delta (B.1.617.2)] or a partial immune escape with variable effects on neutralization by polyclonal serum antibodies [ $\mathrm{VoCs}$ beta (B.1.351), gamma (P.1/B.1.1.28) and delta] [2-7]. A striking characteristic of the apparently independently evolved omicron $\mathrm{VoC}$ is a large number of amino acid substitutions, insertions and deletions in the spike protein, i.e. 32 changes compared to the original Wuhan-hu-1 virus [8], that likely contribute to its extraordinarily rapid spread in the population. Moreover, the number of epitopes in the spike protein relevant for neutralization are an important determinant of the genetic barrier to viral escape from humoral immunity $[6,9]$. Consequently, physician-scientists have been alerted since its discovery based on the genetic information alone by omicron's potential for a pronounced immune escape. Recent laboratory studies and epidemiological reports have now confirmed this exceptional combination of high 
contagiousity and drastic escape from neutralizing antibodies [10-12]. The resistance to neutralization is most likely due to mutations K417N, N440K, G446S, S477N, T478K, E484A, Q493R, G496S, Q498R, N501Y and Y505H, which are located within or close to the epitopes bound by these antibodies [13].

Here, we report the laboratory-based diagnosis of the first four COVID-19 cases with an omicron infection reported in Germany based on a combination of variant-specific PCRs and next-generation-sequencing using an established nanopore platform.

\section{Materials and methods}

\section{Patients and collection of respiratory samples for testing for SARS-CoV-2}

Respiratory samples (either oropharyngeal gargle or nasopharyngeal swab) were collected in Bio-Speedy vNAT Transfer Tubes (Bioeksen R\&D Technologies, Cat. No. BS-NA-513-100) from patients on November 27, 2021, who had self-reported a positive SARS-CoV-2 PCR result two days after returning from Cape Town, South Africa, following a two-week round trip (patient 1 and 2), or patients arriving in Germany at Munich airport on a flight from Cape Town, South Africa, on November 26, 2021 (patient 3 and 4). For additional patient characteristics and sample details see Table 1.

\section{Quantitative viral load determination}

Samples were quantified for SARS-CoV-2 RNA using the fully-automated Roche cobas ${ }^{\circledR}$ 6800/8800 system (Roche, Mannheim, Germany). For quantification, standard curves were generated in multiple replicates using a commercially available standard for calibration (Instand e.V.) as described previously [14]. Viral loads of samples were calculated as SARS-CoV-2 nucleocapsid gene copy numbers per $1 \mathrm{ml}$ of oropharyngeal gargle.

\section{SARS-CoV-2 VOC-specific PCRs}

Nucleic acids from patients' respiratory samples were extracted using the QIAsymphony DSP Virus/Pathogen Kit (cat. no. 937036) on a QIAsymphony SP instrument or the EZ1 Virus Mini Kit v2.0 (cat. no. 955134) with the EZ1 Advanced XL (Qiagen, Hilden, Germany). Eluates were used for melting curve analysis performed on a LightCycler480 II (Software version 1.5.1.62) (Roche Diagnostics, Basel, Switzerland) with the Luna ${ }^{\circledR}$ Probe One-Step RT-qPCR Kit (No ROX) (cat. no. E3007E, NEB, Frankfurt am Main, Germany) and the following 
VirSNiP kits (TIB MOLBIOL, Berlin, Germany): SARS del69/70 + $484 \mathrm{~K}+501 \mathrm{Y}$ (Cat. No. 53-0799-96) (Fig. 1A, top panel), SARS Spike 417 T 681H (Cat. No. 53-080796) (Fig. 1A, middle panel) and SARS Spike S371L S373P (53-0827-96) (Fig. 1a, lower panel). We followed the manufacturer instructions for the VirSNiP assay except adapting the thermocycler program to the Luna ${ }^{\circledR}$ Probe OneStep RT-qPCR mix with the following modification: we shortened the $60{ }^{\circ} \mathrm{C}$ step to $15 \mathrm{~s}$ and added a step at $72{ }^{\circ} \mathrm{C}$ for $15 \mathrm{~s}$ in every cycle. Seegene Variants I and II assays (cat. no. RV10286X and RV10305X) were performed on C1000 Thermal Cyclers (Bio-Rad, Feldkirchen, Germany) according to the manufacturer's instructions.

\section{SARS-CoV-2 whole-genome sequencing}

Sequencing libraries were prepared according to the ARTIC network nCoV-2019 sequencing protocol v3 $[15,16]$. cDNA was generated from extracted total RNA using LunaScript RT (NEB, Ipswich, USA) and amplified with ARTIC primer set v3 [17]. The v3 primer set was rebalanced to achieve a more uniform amplicon coverage [18] and supplemented with updated versions of primer 64_left (ATGTCGATAGATATCCTGTTAATTCCATTG T) and 72_right (CTAGAATAAACTCCACTTTCCATC CAAC) to avoid lineage-specific dropouts. The library quality was routinely checked in each run using standardised control samples. PCR amplification was as described $[15,16]$ with 35 cycles and $64{ }^{\circ} \mathrm{C}$ annealing temperature. PCR reactions were diluted 1:10 and the two primer pool reactions for each sample were pooled. Diluted amplicons were end-repaired, purified with Ampure XP beads, ligated to barcode adapters (Oxford Nanopore Technologies (ONT), Oxford, UK), purified again with Ampure XP beads, pooled and ligated to AMII sequencing adapter (ONT, Oxford, UK). The purified library was sequenced on a PromethION R9.4.1 flowcell until sufficient coverage for lineage calling was reached, monitored by RAMPART software package [19].

Sequencing reads were basecalled with the Guppy basecaller (v.5.0.17) using the super-accurate DNA model provided by ONT (Oxford, UK). The samples were demultiplexed according to the EXP-NBD196 barcode kit. The sequenced amplicons were assembled using the ARTIC bioinformatics protocol [20]. Following the protocol, the artic tool was used to filter the reads by the quality and by a read length between 380 and 700 nucleotides. Reads were mapped to the Sars-Cov-2 reference genome (NC_045512.2) to call variants. Called variants were integrated into the reference sequence to obtain the consensus sequence of the sequenced sample. The lineage assignment was performed with the pangolin tool (v.3.1.16) [21].

\section{Results and discussion}

In four individuals returning from South Africa on November 24 or 26, 2021 to Munich, Germany, SARS-CoV-2 was detected in respiratory swabs by PCR on November 27, 2021 with cycle-threshold (ct) values ranging from 23.2 to 31.2 (Table 1). Spike-gene dropouts (or spike-gene target failure, SGTF) in conventional PCR systems, which can present either as a markedly delayed $\mathrm{Ct}$ value for or an entirely absent detection of the SARS-CoV-2 spike gene, have been reported for B.1.1.529 over the last weeks [22-24] as indirect diagnostic evidence for its identification. Typically, non-spike gene-targeted sequences, e.g. in the nucleocapsid gene, envelope gene or RNA-dependent $R N A$ polymerase gene [25], performed in these omicroncontaining samples in parallel, can be readily amplified.

High-resolution probe-based melting curve assays [26] and multiplex-based PCR assays [27, 28] have allowed simultaneous detection of discriminatory mutation sites for identification and differentiation of VOCs alpha [B.1.1.7], beta [B.1.351], gamma [P.1], and delta [B.1.617.2 and AY]. These tests' applicability to the identification of omicron [B.1.1.529] had not been reported at the time of this study in late November 2021.

Based on the patients' recent travel history to South Africa and available sequences for B.1.1.529 from GISAID [29], we employed SARS-CoV-2 variant-specific PCR systems (Assays 1 and 2) from two globally operating commercial vendors (TIB MOLBIOL, Berlin, Germany, and Seegene, Germany, Düsseldorf, Germany) [26-28], which had at the time either not been officially launched or only recently been launched, on November 27, 2021: RT-PCR products of samples from patients 1-3 (Table 1) showed a peak for del 69/70 (like alpha; unlike beta, gamma and delta) and a shifted and dampened peak for 484 compared to the other VOCs (Fig. 1a (top panel), Tables 2, and 3). The peak for 501 was lost, likely due to surrounding mutations (G496S, Q498R, Y505H). A peak was detected for $417 \mathrm{~N}$ (like beta; unlike alpha, gamma and delta), and a shifted and dampened peak for $681 \mathrm{H}$ (Fig. 1a (middle panel), Tables 2, and 3) (unlike all other VOCs), the latter likely due to omicron's N679K mutation. Also, a distinct melting curve for 371L/373P compared to alpha, beta and delta was observed (Fig. 1a (lower panel), Tables 2, and 3) [26]. Results for the sample from patient 4 were indeterminate in both assays (Fig. 1b), likely due to the very low virus load in this respiratory sample (ct value of 31.3 or negative; Table 1). Figure $1 \mathrm{~b}$ summarizes the results for the VOC-specific assays for omicron (see Table 3 for primary output data for Assay 2 for all VOCs [27, 28]).

Next, RNA extracts of patients' samples were studied by Nanopore sequencing using the ARTIC protocol version 3 
Fig. 1 Detection of omicron (B.1.1.529) by SARS-CoV-2 variant-specific PCRs for the spike gene. a PCR probe-based melting curve assays (ref 26) for the identification of SARSCoV-2 VOCs. Melting curves for spike mutations/deletions at the indicated amino acid positions. Data for all five VOCs are depicted. b Summary of mutational pattern for omicron detected by two different VOC-specific assays (Assay

1: TIB MOLBIOL (ref 26);

Assay 2: Seegene Variants I/ II (refs 27, 28). ${ }^{\dagger}$ Melting curve peak shifted and dampened.

\#Delayed Ct value a

SARS del69/70 + 484K + 501Y

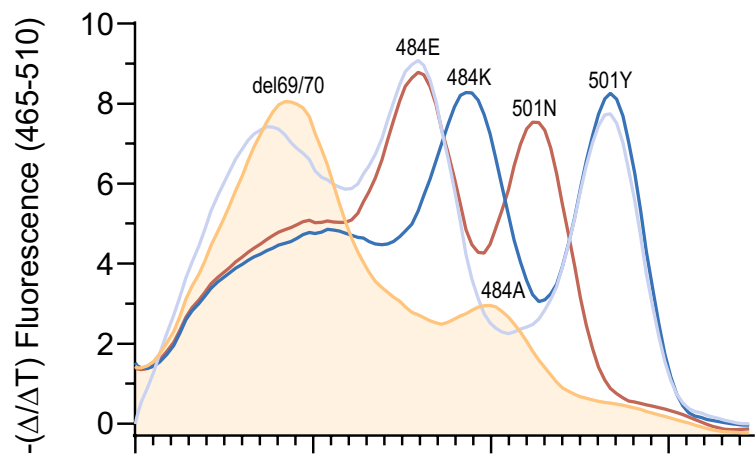

SARS Spike 417T 681H

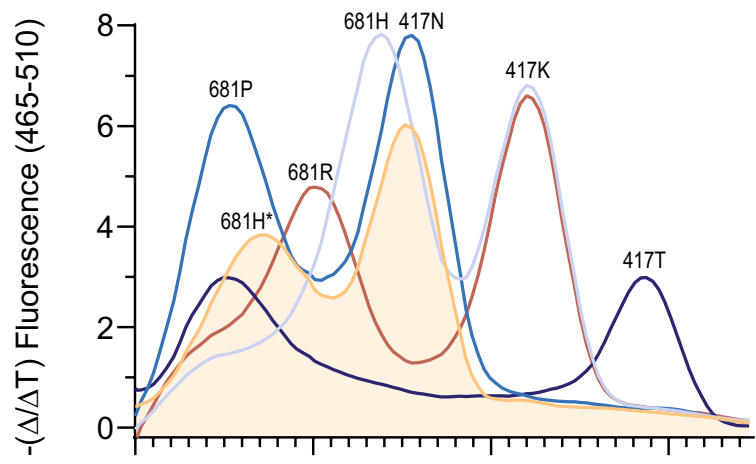

SARS Spike S371L S373P

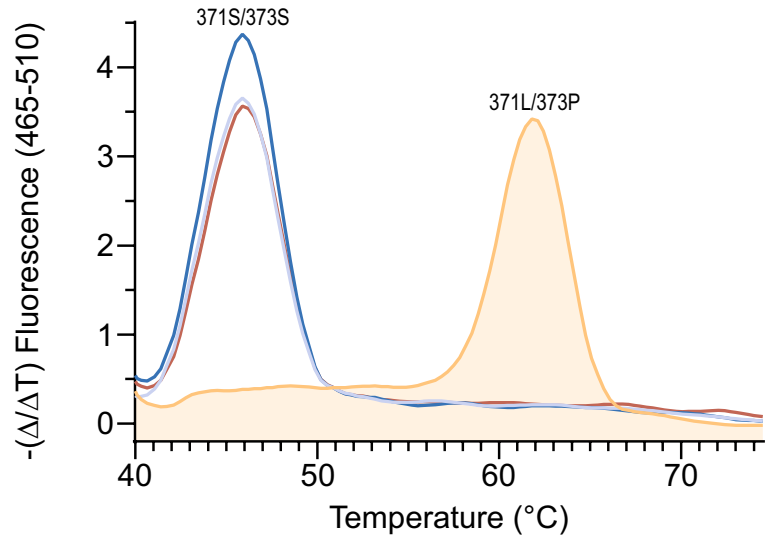

b Omicron spike antigen - mutational pattern

\begin{tabular}{|c|c|c|c|c|c|c|}
\hline & $\begin{array}{c}\text { del } \\
69 / 70 \\
\end{array}$ & $417 \mathrm{~N}$ & $\begin{array}{l}371 \mathrm{~L} \\
373 \mathrm{P} \\
\end{array}$ & $484 \mathrm{~K}$ & $501 Y$ & $681 \mathrm{H}$ \\
\hline Assay 1 & pos & pos & pos & $\operatorname{pos}^{\dagger}$ & neg & pos $^{\dagger}$ \\
\hline ssay 2 & pos & $\operatorname{pos}^{\#}$ & & neg & pos & \\
\hline
\end{tabular}


Table 2 Summary of amino acid mutations in spike relevant for VOC identification

\begin{tabular}{llllllll}
\hline & 69/70 & 417 & 452 & 484 & 501 & 681 & $371 / 373$ \\
\hline Omicron & del & N & L & A & $\mathrm{Y}^{*}$ & $\mathrm{H}^{\#}$ & $\mathrm{~L} / \mathrm{P}$ \\
Alpha & del & $\mathrm{K}$ & $\mathrm{L}$ & $\mathrm{E}$ & $\mathrm{Y}$ & $\mathrm{H}$ & $\mathrm{S} / \mathrm{S}$ \\
Beta & $\mathrm{H} / \mathrm{V}$ & $\mathrm{N}$ & $\mathrm{L}$ & $\mathrm{K}$ & $\mathrm{Y}$ & $\mathrm{P}$ & $\mathrm{S} / \mathrm{S}$ \\
Gamma & $\mathrm{H} / \mathrm{V}$ & $\mathrm{T}$ & $\mathrm{L}$ & $\mathrm{K}$ & $\mathrm{Y}$ & $\mathrm{P}$ & $\mathrm{S} / \mathrm{S}$ \\
Delta & $\mathrm{H} / \mathrm{V}$ & $\mathrm{K}$ & $\mathrm{R}$ & $\mathrm{E}$ & $\mathrm{N}$ & $\mathrm{R}$ & $\mathrm{S} / \mathrm{S}$ \\
\hline
\end{tabular}

Amino acid positions in the SARS-CoV-2 spike protein critical for the identification of VOCs alpha, beta, gamma, delta and omicron are depicted

* Melting curve peak not detected in assay A

\#Peak shifted due to surrounding mutations

\begin{tabular}{llllllll}
\hline & del69/70 & $417 \mathrm{~N}$ & $452 \mathrm{R}$ & $484 \mathrm{~K}$ & $501 \mathrm{Y}$ & $681 \mathrm{H}$ & $\mathrm{S} 371 \mathrm{~L} / \mathrm{S} 373 \mathrm{P}$ \\
\hline Omicron & $\checkmark$ & $\checkmark$ & $\times$ & $\checkmark^{*}$ & $\times$ & $\checkmark *$ & $\checkmark$ \\
Alpha & $\checkmark$ & $\times$ & $\times$ & $\times$ & $\checkmark$ & $\checkmark$ & $\times$ \\
Beta & $\times$ & $\checkmark$ & $\times$ & $\checkmark$ & $\checkmark$ & $\times$ & $\times$ \\
Gamma & $\times$ & $\times$ & $\times$ & $\checkmark$ & $\checkmark$ & $\times$ & $\times$ \\
Delta & $\times$ & $\times$ & $\checkmark$ & $\times$ & $\times$ & $\times$ & $\times$ \\
\hline
\end{tabular}

*Peak shifted and/or dampened due to surrounding mutations
Table 3 Summary of diagnostic findings for spike mutations relevant for VOC identification nanopore-based full-length genome sequencing for the rapid and sensitive identification of the first four COVID19 patients diagnosed in Germany to be infected with omicron. These findings will assist unambiguous laboratorybased clinical diagnosis and global surveillance for this highly contagious VOC, which carries spike mutations conferring reduced COVID-19 vaccine efficacy. Moreover, specialized diagnostic laboratories should continuously update their assays for variant-specific PCRs in the spike gene of SARS-CoV-2 to readily detect and diagnose emerging variants of interest and VoCs. The combination with established nanopore sequencing procedures allows both the rapid confirmation by whole genome sequencing as well as the sensitive identification of newly emerging variants of this pandemic $\beta$-coronavirus in years to come.

Acknowledgements We are grateful to members of the Diagnostic Laboratory of Virology at the Max von Pettenkofer Institute for support. We would like to thank the Bavarian SARS-CoV-Public Health Laboratory Epidemiology Team at the LGL for support.

Author contributions OTK designed the study. CD, MM, HA, SB, HM, ST and SK performed experiments. CD, MM, AG, PRW, HM NG-K, HB and OTK analyzed data. CD and PRW assembled graphics. CD, MM and OTK wrote the manuscript. All authors discussed the results and commented on the final manuscript.

Funding Open Access funding enabled and organized by Projekt DEAL. This work was supported in part by the German BMBF initiative "NaFoUniMedCovid19" (01KX2021), subproject B-FAST (to O.T.K.), and by the Free State of Bavaria (research initiatives Bay-VOC (M.M., A.G., S.K., H.B., O.T.K.) and FOR-COVID (M.M., O.T.K.).
In conclusion, we report the combination of PCRs specific for heavily mutated sites in the spike gene and 
Availability of data and material Not applicable.

Code availability Not applicable.

\section{Declarations}

Conflict of interest The authors declare that they have no conflict of interest.

Ethics approval The generation and publication of SARS-CoV-2 sequences and accompanying metadata were approved by the local ethics committee at the Medical Faculty of LMU München (No. 21-0740).

Open Access This article is licensed under a Creative Commons Attribution 4.0 International License, which permits use, sharing, adaptation, distribution and reproduction in any medium or format, as long as you give appropriate credit to the original author(s) and the source, provide a link to the Creative Commons licence, and indicate if changes were made. The images or other third party material in this article are included in the article's Creative Commons licence, unless indicated otherwise in a credit line to the material. If material is not included in the article's Creative Commons licence and your intended use is not permitted by statutory regulation or exceeds the permitted use, you will need to obtain permission directly from the copyright holder. To view a copy of this licence, visit http://creativecommons.org/licenses/by/4.0/.

\section{References}

1. Mohapatra RK, Sarangi AK, Kandi V et al (2021) Omicron (B.1.1.529 variant of SARS-CoV-2); an emerging threat: current global scenario. J Med Virol. https://doi.org/10.1002/jmv.27561

2. Volz E, Mishra S et al (2021) Assessing transmissibility of SARSCoV-2 lineage B.1.1.7 in England. Nature 593:266-269. https:// doi.org/10.1038/s41586-021-03470-x

3. Tegally H, Wilkinson E, Giovanetti M et al (2021) Detection of a SARS-CoV-2 variant of concern in South Africa. Nature 592:438443. https://doi.org/10.1038/s41586-021-03402-9

4. Fujino T, Nomoto H, Kutsuna S et al (2021) Novel SARS-CoV-2 variant in travelers from Brazil to Japan. Emerg Infect Dis. https:// doi.org/10.3201/eid2704.210138

5. Mlcochova P, Kemp SA, Dhar MS et al (2021) SARS-CoV-2 B.1.617.2 delta variant replication and immune evasion. Nature 599:114-119. https://doi.org/10.1038/s41586-021-03944-y

6. Wang Z, Schmidt F, Weisblum Y et al (2021) mRNA vaccine-elicited antibodies to SARS-CoV-2 and circulating variants. Nature 592:616-622. https://doi.org/10.1038/s41586-021-03324-6

7. Planas D, Veyer D, Baidaliuk A et al (2021) Reduced sensitivity of SARS-CoV-2 variant Delta to antibody neutralization. Nature 596:276-280. https://doi.org/10.1038/s41586-021-03777-9

8. Karim SSA, Karim QA (2021) Omicron SARS-CoV-2 variant: a new chapter in the COVID-19 pandemic. Lancet 398:2126-2128. https://doi.org/10.1016/S0140-6736(21)02758-6

9. Schmidt F, Weisblum Y, Rutkowska M et al (2021) High genetic barrier to SARS-CoV-2 polyclonal neutralizing antibody escape. Nature 600:512-516. https://doi.org/10.1038/s41586-021-04005-0

10. Collie S, Champion J, Moultrie H et al (2021) Effectiveness of BNT162b2 vaccine against omicron variant in South Africa. N Engl J Med. https://doi.org/10.1056/NEJMc2119270

11. Nemet I, Kliker L, Lustig Y et al (2021) Third BNT162b2 vaccination neutralization of SARS-CoV-2 omicron infection. N Engl J Med. https://doi.org/10.1056/NEJMc2119358
12. Gruell H, Vanshylla K, Tober-Lau P et al (2021) mRNA booster immunization elicits potent neutralizing serum activity against the SARS-CoV-2 Omicron variant. Res Square. https://doi.org/ 10.21203/rs.3.rs-1168453/v1

13. Hoffmann M, Krüger N, Schulz S et al (2022) The omicron variant is highly resistant against antibodymediated neutralizationimplications for control of the COVID-19 pandemic. Cell. https:// doi.org/10.1016/j.cell.2021.12.032

14. Osterman A, Baldauf H-M, Eletreby M et al (2021) Evaluation of two rapid antigen tests to detect SARS-CoV-2 in a hospital setting. Med Microbiol Immunol 210:65-72. https://doi.org/10. 1007/s00430-020-00698-8

15. Quick J (2020) nCoV-2019 sequencing protocol v3 (LoCost) V.3. In: protocols.io. https://protocols.io/view/ncov-2019-sequencingprotocol-v3-locost-bh42j8ye. Accessed 04 Jan 2022

16. Tyson JR, James P, Stoddart D et al (2020) Improvements to the ARTIC multiplex PCR method for SARS-CoV-2 genome sequencing using nanopore. BioRxiv. https://doi.org/10.1101/ 2020.09.04.283077

17. Quick J (2020) artic-ncov2019. https://github.com/joshquick/ artic-ncov2019/blob/master/primer_schemes/nCoV-2019/V3/ nCoV-2019.tsv. Accessed 4 Jan 2022

18. DNA Pipelines R\&D, Farr B, Rajan D, et al (2020) COVID-19 ARTIC v3 Illumina library construction and sequencing protocol. In: protocols.io. https://www.protocols.io/view/covid-19-artic-v3illumina-library-construction-an-bibtkann. Accessed 4 Jan 2022

19. Artic Network (2020) rampart. https://github.com/artic-network/ rampart. Accessed 4 Jan 2022

20. Artic Network. https://artic.network/ncov-2019

21. O'Toole Á, Scher E, Underwood A et al (2021) Assignment of epidemiological lineages in an emerging pandemic using the pangolin tool. Virus Evol. https://doi.org/10.1093/ve/veab064

22. Classification of Omicron (B.1.1.529): SARS-CoV-2 Variant of Concern. https://www.who.int/news/item/26-11-2021-class ification-of-omicron-(b.1.1.529)-sars-cov-2-variant-of-concern. Accessed 04 Jan 2022

23. European Centre for Disease Prevention and Contro (2021) Implications of the emergence and spread of the SARS-CoV-2 B.1.1. 529 variant of concern (Omicron) for the EU/EEA

24. Thermo Fisher Scientific Confirms Detection of SARS-CoV-2 in Samples Containing the Omicron Variant with its TaqPath COVID-19 Tests. In: MediaRoom. https://thermofisher.media room.com/2021-11-29-Thermo-Fisher-Scientific-Confirms-Detec tion-of-SARS-CoV-2-in-Samples-Containing-the-Omicron-Varia nt-with-its-TaqPath-COVID-19-Tests. Accessed 4 Jan 2022

25. Muenchhoff M, Mairhofer H, Nitschko H, Grzimek-Koschewa N, Hoffmann D, Berger A, Rabenau H, Widera M, Ackermann N, Konrad R, Zange S, Graf A, Krebs S, Blum H, Sing A, Liebl B, Wolfel R, Ciesek S, Drosten C, Protzer U, Boehm S, Keppler OT (2020) Multicentre comparison of quantitative PCR-based assays to detect SARS-CoV-2, Germany, March 2020. Euro Surveill. https://doi.org/10.2807/1560-7917.ES.2020.25.24.2001057

26. Covid-19-TIB MOLBIOL. https://www.tib-molbiol.de/covid-19. Accessed 4 Jan 2022

27. Seegene Allplex SARS-CoV-2 Variants I Assay. https://www. seegene.com/assays/allplex_sars-cov-2_variants_i_assay. Accessed 4 Jan 2022

28. Seegene Allplex SARS-CoV-2 Variants II Assay. https://www. seegene.com/assays/allplex_sars-cov-2_variants_ii_assay. Accessed 4 Jan 2022

29. Cov-Lineages. https://cov-lineages.org/global_report_B.1.1.529. html. Accessed 4 Jan 2022

30. SARS-CoV-2 V4.1 update for Omicron variant. https://commu nity.artic.network/t/sars-cov-2-v4-1-update-for-omicron-variant/ 342). Accessed 7 Jan 2022 
31. Bull RA, Adikari TN, Ferguson JM et al (2020) Analytical validity of nanopore sequencing for rapid SARS-CoV-2 genome analysis. Nat Commun 11:6272. https://doi.org/10.1038/ s41467-020-20075-6

Publisher's Note Springer Nature remains neutral with regard to jurisdictional claims in published maps and institutional affiliations. 